\title{
Nephritogenic Antigen Determinants in Epidermal and Renal Basement Membranes of Kindreds with Alport-type Familial Nephritis
}

\author{
Clifford Kashtan, Alfred J. Fish, Mary Kleppel, Kazuo Yoshioka, and Alfred F. Michael \\ Departments of Pediatrics, Laboratory Medicine, and Pathology, University of Minnesota Medical School, Minneapolis, Minnesota 55455
}

\begin{abstract}
We probed epidermal basement membranes (EBM) of acid-urea denatured skin from members of kindreds with Alport-type familial nephritis (FN) for the presence of antigens reactive with Goodpasture sera (GPS) and serum (FNS) from an Alport patient who developed anti-glomerular basement membrane (GBM) nephritis in a renal allograft. By immunoblotting, GPS reacted primarily with the 28,000 molecular weight (mol wt) monomer but also the $24,000 \mathrm{~mol}$ wt and $26,000 \mathrm{~mol}$ wt monomers of the noncollagenous globular domain (NC1) of type IV collagen from normal human GBM, while FNS identified only the $26,000-\mathrm{mol}$ wt monomer.

FNS reacted with EBM of 12 controls and nine unaffected male kindred members but not EBM of eight affected males. Five affected females exhibited interrupted reactivity of FNS with EBM. GPS showed variable reactivity with EBM and was not discriminating with respect to Alport-type FN. FNS did not stain renal basement members of five affected males. However, the EBM, tubular basement membrane, and Bowman's capsules of affected males contained antigens reactive with GPS. These immunochemical studies suggest that the FNS antigen is distinct from Goodpasture antigen(s).

The expression of FNS antigen located on the NC1 domain of type IV collagen is altered in basement membranes of patients with Alport-type FN, and the distribution of this antigenic anomaly within kindreds suggests $\mathrm{X}$-linked dominant transmission of a defective gene.
\end{abstract}

\section{Introduction}

Alport-type familial nephritis $(\mathrm{FN})^{1}$ is a hereditary disorder characterized by progressive renal insufficiency (predominantly

Portions of this work were presented at the American Society of $\mathrm{Ne}$ phrology, New Orleans, LA, 18 December 1985.

Dr. Kashtan is a U. S. Public Health Services postdoctoral fellow (AM07087). Dr. Yoshioka's present address is Department of Pediatrics, Kinki University School of Medicine, Sayama, Minamikawachi, Osaka 589, Japan. Address reprint requests to Dr. Michael, Department of Pediatrics, University of Minnesota, Box 491 Mayo, 420 Delaware St. S.E., Minneapolis, MN 55455.

Received for publication 20 November 1985 and in revised form 17 April 1986.

1. Abbreviations used in this paper: AGBM, serum from a patient with idiopathic anti-glomerular basement membrane nephritis; BFH, benign familial hematuria; $\mathrm{C} 3$, third component of human complement; $\mathrm{EBM}$,

J. Clin. Invest.

(C) The American Society for Clinical Investigation, Inc.

0021-9738/86/10/1035/10 \$1.00

Volume 78, October 1986, 1035-1044 in affected males), sensorineural hearing loss, and in some families, ocular defects such as anterior lenticonus. Ultrastructural (1-8) studies have demonstrated abnormalities in the glomerular basement membrane (GBM). While tubular basement membranes (TBM) and Bowman's capsule may exhibit similar alterations (9), little is known about extrarenal basement membranes in this disorder.

The ultrastructural GBM anomalies are accompanied, at least in some patients, by apparent alterations in the antigenicity of the GBM. After renal transplantation, some patients with Alporttype FN have developed anti-GBM nephritis in their renal allografts (10-12). In addition, the IgG in sera from patients with Goodpasture's syndrome, which binds in vitro to normal GBM as assessed by indirect immunofluorescence, does not bind to the GBM of some patients with Alport-type FN (10, 13-16).

Previous work from this laboratory (17) has demonstrated that denaturation of various human tissues in acid-urea uncovers antigenic determinants in basement membranes and enhances reactivity with human anti-GBM antibodies, as revealed by indirect immunofluorescence. In the present study we employed this methodology to investigate the distribution of nephritogenic antigens in renal and epidermal basement membranes in kindreds with Alport-type FN. The probes consisted of three sera from patients with Goodpasture's syndrome (GPS1, GPS2, and GPS3), serum from a patient with idiopathic anti-GBM nephritis (AGBM), and serum from a patient with Alport-type FN (FNS) who developed anti-GBM nephritis in a renal allograft. We found that epidermal basement membranes of Alport patients exhibited antigenic anomalies similar to those found in their renal basement membranes and that these antigenic changes were distributed within kindreds in a pattern consistent with Xlinked dominant transmission of a defective gene. The antigen reactive with GPS was present in at least some Alport basement membranes, although these same basement membranes apparently lacked an antigen(s) reactive with FNS. In addition, we employed immunochemical methods to characterize the reactivity of these probes with monomeric and dimeric components of the noncollagenous globular domain of type IV collagen (NC1), in which the epitopes reactive with human anti-GBM antibodies are located (18).

\section{Methods}

Type VII collagenase, 3-3' diaminobenzidine (acid form), phenylmethylsulfonyl fluoride (PMSF), $N$-ethylmaleimide (NEM), pepstatin A, and

epidermal basement membrane; ELISA, enzyme-linked immunosorbent assay; FITC, fluorescein-isothiocyanate; FN, familial nephritis; FNS, serum from a patient with Alport-type FN; GBM, glomerular basement membrane; GPS1, 2, and 3, three sera from patients with Goodpasture's syndrome; $\mathrm{NC1}$, noncollagenous globular domain of type IV collagen; NEM, $N$-ethylmaleimide; NHS, normal human serum; PMSF, phenylmethylsulfonyl fluoride; SDS-PAGE, sodium dodecyl sulfate-polyacrylamide gel electrophoresis; TBM, tubular basement membrane. 
bovine serum albumin (BSA) were obtained from Sigma Chemical Co. (St. Louis, MO). Low molecular weight standards for electrophoresis were purchased from Pharmacia Fine Chemicals (Piscataway, NJ). Peroxidase-labeled, affinity-purified sheep anti-human $\operatorname{IgG~} \mathrm{F}\left(\mathrm{ab}^{\prime}\right)_{2}$, fluorescein-isothiocyanate (FITC)-conjugated rabbit anti-human IgG, FITCconjugated goat anti-mouse IgG, and rhodamine-labeled goat anti-mouse IgG were obtained from Cappel Laboratories (Cochranville, PA). Rabbit antiserum to mouse (EHS sarcoma) laminin was obtained from Bethesda Research Laboratories (Gaithersburg, MD), FITC-labeled goat anti-rabbit IgG was purchased from Kallestad Laboratories, Inc. (Austin, TX). The characteristics of mouse monoclonal antibody to human type IV collagen have been reported (19).

\section{Patients and controls}

Skin specimens (3-5 $\mathrm{mm}$ in diameter) were obtained by punch biopsy from the volar aspect of the forearm from members of four kindreds with familial nephritis of the Alport type (families A-D). The diagnosis of Alport-type FN was made when a family satisfied all of the following criteria: (a) the finding of hematuria in multiple members of a family; (b) progression to renal insufficiency in at least one male kindred member; (c) the presence of progressive sensorineural hearing loss in males with renal disease; and $(d)$ the finding, in at least one affected male in a kindred, of diffuse splitting and multilamination of the GBM by electron microscopy, along with negative direct immunofluorescence. These families exhibited inheritance patterns compatible with autosomal dominant or $\mathrm{X}$-linked dominant transmission; there were no instances of male-tomale transmission of the disorder. Any member of a kindred who demonstrated hematuria on multiple urinalyses was considered affected. Sensorineural hearing loss was documented by audiometry in kindred members with clinically apparent hearing deficits; we did not perform audiometry on all kindred members. The pedigrees of families A-D are presented in Fig. 1. Families A-D were the subjects of a previous publication (15).

We included in our studies three affected members of two kindreds ( $\mathrm{E}$ and $\mathrm{F}$ ) with benign familial hematuria (BFH). The diagnosis of BFH rested on the following criteria: $(a)$ the finding of hematuria in multiple members of a family; $(b)$ the absence of renal insufficiency in the proband or, by history, in any family members; and $(c)$ the finding in the proband, by electron microscopy of diffuse thinning of the GBM without splitting, multilamination, or electron-dense deposits. In addition, direct immunofluorescence studies of renal biopsy material for immunoglobulin and complement components were negative.

A seventh family satisfied all the criteria for the diagnosis of Alporttype FN and was similar to kindreds A-D above, but electron microscopic examination of renal tissue was not performed. For the latter reason, this kindred is not included as a lettered family in the tabulated data. However, we mention this family in this study because skin specimens obtained from three affected members (two males and one female) showed a pattern of reactivity with FNS that was different from that observed in affected members of kindreds A-D, as described in Results.

Renal biopsy material was available for indirect immunofluorescence studies from five affected males in kindreds A-D. This tissue was obtained by percutaneous biopsy during clinical evaluation, or by nephrectomy at the time of renal transplantation. At the time renal tissue was obtained, renal function was normal in three subjects (A-II-2, C-II-3, and D-III-1) and end-stage in two subjects (B-II-1 and C-II-2). Subjects A-II-1, D-III4, D-III-5, and D-III-7 had undergone renal biopsy; however, renal tissue was not available for indirect immunofluorescence studies in these cases. Renal tissue obtained from two males (kindreds $E$ and $F$ ) with BFH was also available for indirect immunofluorescence studies.

Specimens of skin were also obtained from 12 control individuals (five males, seven females). Four of the normal skin specimens were obtained from volunteers, and eight specimens were obtained during the course of clinical evaluation of various disorders. The latter were histologically and immunohistochemically normal. Normal kidney tissues were obtained by kidney biopsy from a patient as part of an evaluation for kidney donation, and at the time of surgery from a patient with metastatic carcinoma, a patient with renal pelvic disruption, and a patient with an unspecified renal mass.

The protocol for collection and study of skin specimens from patients, family members, and controls was approved by the Committee on the Use of Human Subjects in Research of the University of Minnesota, Minneapolis, MN. Informed consent was obtained from each individual studied.

\section{Characterization of anti-GBM sera ${ }^{2}$}

GPS1, GPS2, and GPS3 were obtained from three patients with Goodpasture's syndrome; a fourth serum (AGBM) was obtained from a patient with anti-GBM nephritis in the absence of pulmonary hemorrhage. FNS was obtained from a patient with Alport-type familial nephritis who developed severe anti-GBM antibody-mediated glomerulonephritis after renal transplantation and is denoted B-II-1 in Fig. 1; the kidney donor was his HLA-identical brother (B-II-2). Light microscopy of the allograft revealed a severe crescentic glomerulonephritis, and immunohistochemical studies demonstrated extensive linear deposition of IgG, IgA, and third component of human complement (C3) along the GBM. IgG and C3 were noted focally along tubular basement membranes (TBM) as well.

Anti-GBM activity was determined in the anti-GBM sera by two methods. Indirect immunofluorescence showed titers of $>1: 64$ for GPS1, GPS3, AGBM, and FNS, while GPS2 had a titer of 1:4. Using an enzymelinked immunosorbent assay (ELISA) for anti-GBM antibodies (21), titers of $>1: 160,1: 40,1: 80,1: 160$, and 1:40 were obtained for GPS1, GPS2, GPS3, AGBM, and FNS, respectively.

Reactivities of GPS1, GPS2, GPS3, AGBM, and FNS with isolated components of the globular noncollagenous domain (NC1) of type IV collagen were also examined. The following techniques for extraction, separation, and identification of these components were employed.

Isolation of GBM. Normal human kidneys were provided by the National Diabetes Research Interchange. These kidneys were harvested from patients with traumatic deaths but were not used for transplantation.

Glomeruli were isolated on ice in phosphate-buffered saline (PBS) with protease inhibitors (PMSF and NEM) as previously described (22). GBM was obtained by ultrasonic disruption in $1 \mathrm{M} \mathrm{NaCl}$ of whole glomeruli which had been separated by size-selective sieving of minced cortex. The sonicated GBM was passed through a No. 300 mesh screen to remove Bowman's capsules and tubular debris, washed three times in PBS with inhibitors, and once with distilled water before lyophilization.

$G B M$ extraction. The lyophilized GBM was extracted twice at $4^{\circ} \mathrm{C}$ with $0.5 \mathrm{M} \mathrm{NaCl}$ in PBS with inhibitors (PMSF and NEM) for $24 \mathrm{~h}$. The GBM was then extracted for $72 \mathrm{~h}$ in $3 \%$ acetic acid containing inhibitors (PMSF, NEM, and pepstatin A) at $4^{\circ} \mathrm{C}$. The salt and acidextracted GBM was then washed three times with distilled water and lyophilized (23).

Collagenase digestion. The extracted GBM was digested with bacterial collagenase $\left(10 \mathrm{U} / \mathrm{mg}\right.$ protein) at $37^{\circ} \mathrm{C}$ for $24 \mathrm{~h}$. The solubilized material was collected by centrifugation at $11,000 \mathrm{~g}$, dialyzed twice against ammonium bicarbonate, $\mathrm{pH} 7.8$, and once against distilled water, and lyophilized (20).

Electrophoretic analysis. Sodium dodecyl sulfate (SDS)-polyacrylamide gel electrophoresis (PAGE) of collagenase digests of GBM was performed in a slab gel electrophoresis unit (Bio-Rad Laboratories, Richmond, CA), using 8-18\% linear gradient gels in a discontinuous buffer system (24). Samples of digested GBM were applied using 20-40 $\mu \mathrm{g} /$ lane. Low molecular weight standards dissolved in SDS sample buffer were used at $9 \mu \mathrm{g} / \mathrm{lane}$. Gels were stained for protein with Coomassie Blue dye.

Immunochemistry. Immunoblotting was carried out using the method of Burnette (25). Proteins were electrophoretically transferred from SDS-

2. GPS1, GPS2, GPS3, AGBM, and FNS are sera numbers $18,19,11$, 5 and 2, respectively, from a panel of stored anti-GBM sera available in this laboratory (20). 
PAGE gels to $0.45-\mu \mathrm{m}$ nitrocellulose sheets (26), using 200-mA constant current at $4^{\circ} \mathrm{C}$ for $2 \mathrm{~h}$. The blotted proteins were exposed to anti-GBM sera at 1:20 dilution in $0.05 \mathrm{M}$ Tris, $0.2 \mathrm{M} \mathrm{NaCl}, 1 \% \mathrm{BSA}, \mathrm{pH} 7.4$, for $1 \mathrm{~h}$ at room temperature. Peroxidase-labeled sheep anti-human IgG $F\left(a b^{\prime}\right)_{2}$ diluted 1:500 in the same buffer served as the secondary antibody. After a 45-min incubation at room temperature and washing, the bound peroxidase-labeled secondary antibody was reacted with 3-3' diaminobenzidine ( $20 \mathrm{mg}$ in $50 \mathrm{ml}$ Tris saline buffer without BSA), in the presence of $0.03 \% \mathrm{H}_{2} \mathrm{O}_{2}$. The reaction was terminated by washing in distilled water.

\section{Immunohistologic techniques}

Indirect immunofluorescence studies were carried out as previously described (17). Kidney and skin specimens were immersed in isopentane precooled in liquid nitrogen, and stored at $-70^{\circ} \mathrm{C}$ until used. The specimens were sectioned in a Lipshaw cryostat in a constant humidity $(30 \%)$ and temperature $\left(22^{\circ} \mathrm{C}\right)$ room. Skin sections were mounted on glass slides coated with chrome-alum gelatin as an adhesive to minimize detachment of tissue during urea incubation (see below), a problem not encountered with renal tissue. Urea denaturation was carried out using the methods described by Brandtzaeg (27) and Bene (28), as previously reported from this laboratory (17). Sections $(4-\mu \mathrm{m})$ of skin or renal cortex were air-dried and fixed in $95 \%$ ethanol at $4^{\circ} \mathrm{C}$ for $5 \mathrm{~min}$, followed by three 3-min rinses in PBS ( $0.145 \mathrm{M}$ sodium chloride, $0.01 \mathrm{M}$ phosphate, $\mathrm{pH}$ 7.35) at room temperature. The sections were then incubated in a 6-M urea-0.1 M glycine solution ( $\mathrm{pH} 3.5$ ) for $1 \mathrm{~h}$ at $4^{\circ} \mathrm{C}$. Urea-incubated and nonincubated sections were washed three times in PBS and then reacted at room temperature with normal human serum (NHS, a pool of six normal human sera provided by the Complement Laboratory of the University of Minnesota Hospitals and used at a dilution of 1:4), GPS1 (1:4), GPS2 (1:4), GPS3 (1:2), AGBM (1:2), or FNS (1:16), followed by FITC-conjugated rabbit anti-human IgG.

Specimens were also stained with a mouse monoclonal antibody to human type IV collagen, diluted 1:50, followed by FITC-labeled goat anti-mouse IgG, or with rabbit antiserum to mouse (EHS sarcoma) laminin, diluted 1:32, followed by FITC-labeled goat anti-rabbit IgG. Dual antibody staining was carried out by reacting the same section of skin sequentially with FNS and FITC-labeled rabbit anti-human IgG followed by mouse anti-type IV collagen and rhodamine-labeled goat anti-mouse IgG. Sections stained with secondary antibodies alone, with and without urea treatment, served as controls. After staining, all sections were treated with $p$-phenylenediamine to retard fading of fluorescence (29).

The slides were viewed under an epifluorescence microscope (Carl Zeiss, Inc., Thornwood, NY) with appropriate filters, by two observers (Dr. Kashtan and Dr. Michael). The intensity of fluorescence of basement membranes was graded as follows: 0 , trace, $1+, 2+, 3+$.

\section{Results}

Immunohistological studies. The results of the immunohistological studies are summarized in Fig. 1 and in Table I. After incubation of normal human skin with FNS, binding of IgG to the epidermal basement membranes (EBM) could be demonstrated consistently; this reactivity was enhanced significantly if the tissue was preincubated with urea (Table I, Figs. 2 and 3). However, binding of FNS IgG was either absent or was no greater than that observed with NHS in urea-treated or untreated skin of the eight affected males in kindreds A-D (Figs. $2 C$ and $3 C$ ). FNS IgG did bind to the EBM of all nine unaffected males in these kindreds, and the intensity of fluorescence was markedly enhanced by urea preincubation (Fig. $2 A$ ).

Seven affected females in kindreds A-D were studied. Six of these females have asymptomatic hematuria, while one (D$\mathrm{I}-2$ ) is in renal failure (serum creatinine, $7.0 \mathrm{mg} / \mathrm{dl}$ ) at age $73 \mathrm{y}$. The latter exhibited only trace reactivity of FNS IgG with EBM
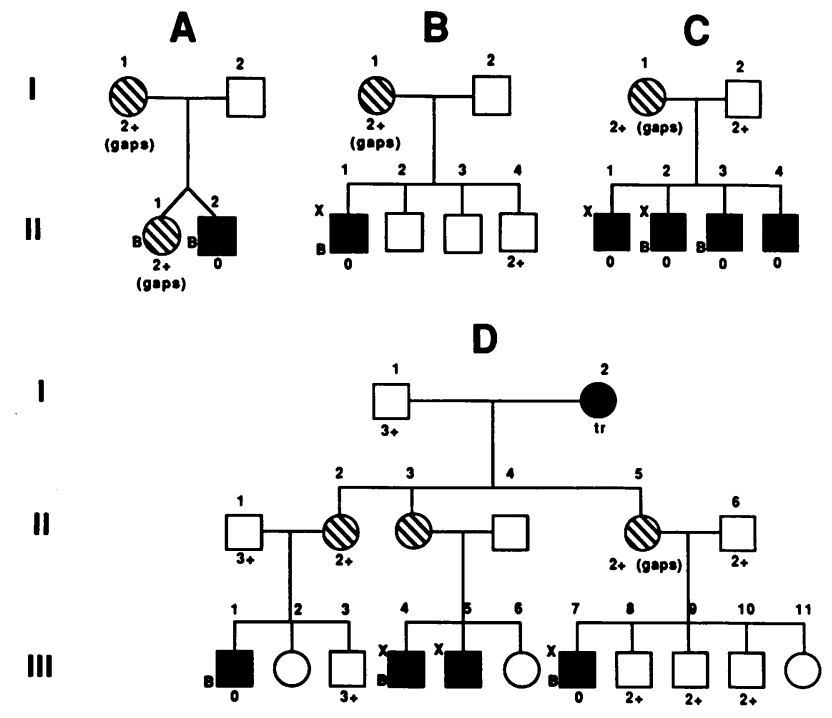

Figure 1. Pedigrees of four kindreds with Alport-type familial nephritis. In these kindreds FNS (serum from patient B-II-1 who developed anti-GBM nephritis after transplantation of a kidney from his HLAidentical sibling, B-II-2) failed to stain epidermal basement membranes (EBM) of urea-denatured skin of males with the disease in comparison to controls or unaffected kindred members. Circles and squares denote affected males and females, respectively. Solid symbols denote individuals with renal disease and sensorineural hearing loss, while cross-hatched symbols indicate individuals with renal involvement alone. $B$ indicates a subject with renal biopsy findings (including electron microscopy) typical of Alport-type familial nephritis. Patients who have received renal transplants are identified by an $X$. Intensity of EBM fluorescence after staining of urea-denatured skin with FNS is described below the circle or square representing a family member. Fluorescence intensity was graded as 0 , trace (tr), $1+, 2+$, and $3+$. Gaps refers to regions of nonreactive EBM separating regions of reactive EBM.

of urea-treated skin. Five of the affected females in these kindreds showed discontinuous binding of FNS IgG to EBM of ureatreated skin, with areas of bright fluorescence separated by lengthy gaps of no reactivity (Fig. $2 \mathrm{~B}$ ). Such gaps in EBM immunofluorescence were observed only in affected female kindred members. That this pattern of reactivity was not an artifact of sectioning or staining was demonstrated by the finding of continuous binding of a mouse monoclonal antibody to type IV collagen (detected with rhodamine-labeled goat anti-mouse IgG) on sections also stained with FNS and FITC-conjugated rabbit anti-human IgG (Fig. 4). In contrast to normal controls or unaffected males, four of the affected females failed to demonstrate binding of FNS IgG to non-urea-treated skin, and in two only trace reactivity was observed. In subjects B-I-1 and D-II-5, interrupted binding of FNS IgG to EBM was observed on nonurea-treated sections as well as on urea-treated sections.

GPS1 was incubated with all skin specimens obtained. GPS2, GPS3, and AGBM were reacted with skin specimens from four affected males (A-II-2, B-II-1, C-II-2, D-III-7), four affected females (A-II-1, B-I-1, C-I-1, D-II-5) and three unaffected males (B-II-4, C-I-2, D-II-6). GPS2 was examined on skin specimens from seven of the controls, while GPS3 and AGBM were reacted with skin from six controls.

GPS1, GPS2, GPS3, and AGBM did not react with EBM 
Table I. Reactivity by Indirect Immunofluorescence of IgG in AGBM Sera with EBM

of Kindreds with Alport-type Familial Nephritis (FN), Benign Familial Hematuria, and Controls*

\begin{tabular}{|c|c|c|c|c|c|c|}
\hline \multirow[b]{2}{*}{ Subjects } & \multirow[b]{2}{*}{ Sex } & \multicolumn{2}{|c|}{ Familial nephritis anti-GBM serum‡ } & \multirow{2}{*}{$\begin{array}{l}\text { Goodpasture sera } \\
\text { (+ Urea\&) GPS1 }\end{array}$} & \multirow{2}{*}{$\begin{array}{l}\text { Anti-type IV } \\
\text { collagen }\end{array}$} & \multirow[b]{2}{*}{ Anti-laminin } \\
\hline & & No urea & +Urea & & & \\
\hline \multicolumn{7}{|c|}{ Alport-type FN } \\
\hline A-I-1 & $\mathbf{F} \mathbb{T}$ & 0 & $2+(\text { gaps })^{\prime \prime}$ & $1+$ & $2+$ & $2+$ \\
\hline A-II-1 & Fq & 0 & $2+$ (gaps) & $1+$ & $2+$ & $1+$ \\
\hline A-II-2 & $\mathbf{M} \pi$ & 0 & 0 & $1+$ & $2+$ & $2+$ \\
\hline B-I-1 & Fq & $1+($ gaps $)$ & $2+$ (gaps) & 0 & $2+$ & $3+$ \\
\hline B-II-1 & Mף & 0 & 0 & $1+$ & $2+$ & $2+$ \\
\hline B-II-4 & $\mathbf{M}$ & $1+$ & $2+$ & $\operatorname{Tr}$ & $2+$ & $3+$ \\
\hline C-I-1 & $\mathbf{F T}$ & 0 & $2+$ (gaps) & $1+$ & $2+$ & $2+$ \\
\hline C-I-2 & $\mathbf{M}$ & $1+$ & $2+$ & $1+$ & $2+$ & $1+$ \\
\hline C-II-1 & $\mathbf{M} \Upsilon$ & 0 & 0 & $1+$ & $2+$ & $1+$ \\
\hline C-II-2 & $\mathbf{M}$ & 0 & 0 & 0 & $2+$ & $2+$ \\
\hline C-II-3 & $\mathbf{M} \pi$ & 0 & 0 & $1+$ & $2+$ & $2+$ \\
\hline C-II-4 & $\mathbf{M} \Upsilon$ & 0 & 0 & $1+$ & $2+$ & $2+$ \\
\hline D-I-1 & $\mathbf{M}$ & $1+$ & $3+$ & $1+$ & $2+$ & $2-3+$ \\
\hline D-I-2 & $\mathbf{F} \mathbb{T}$ & 0 & $\operatorname{Tr}$ & $1+$ & $2+$ & $2-3+$ \\
\hline D-II-1 & $\mathbf{M}$ & $1+$ & $3+$ & $1+$ & $1+$ & $1+$ \\
\hline D-II-2 & $\mathbf{F} \mathbb{1}$ & $\operatorname{Tr}$ & $2+$ & $1+$ & $1+$ & $1+$ \\
\hline D-II-5 & $\mathbf{F} \mathbb{T}$ & $\operatorname{Tr}$ (gaps) & $2+$ (gaps) & $1+$ & $2+$ & $2+$ \\
\hline D-II-6 & $\mathbf{M}$ & $1+$ & $2+$ & $1+$ & $2+$ & $2+$ \\
\hline D-III-1 & MT & 0 & 0 & $1+$ & $2+$ & $1+$ \\
\hline D-III-3 & $\mathbf{M}$ & $1+$ & $3+$ & $1+$ & $2+$ & $2+$ \\
\hline D-III-7 & MT & 0 & 0 & $1+$ & $2+$ & $2+$ \\
\hline D-III-8 & $\mathbf{M}$ & $1+$ & $2+$ & $1+$ & $2+$ & $2+$ \\
\hline D-III-9 & $\mathbf{M}$ & $1+$ & $2+$ & $1+$ & $2+$ & $3+$ \\
\hline D-III-10 & $\mathbf{M}$ & $1+$ & $2+$ & $1+$ & $2+$ & $3+$ \\
\hline \multicolumn{7}{|c|}{ Benign familial hematuria } \\
\hline E-I-2 & $\mathrm{F} \mathbb{1}$ & $1+$ & $2+$ & $1+$ & $2+$ & $2+$ \\
\hline E-II-3 & MT & $\operatorname{Tr}$ & $2+$ & $1+$ & $2+$ & $2+$ \\
\hline F-IV-1 & $\mathbf{M} \pi$ & $1+$ & $2+$ & $1+$ & $2+$ & $2+$ \\
\hline \multicolumn{7}{|l|}{ Controls } \\
\hline$n=5$ & $\mathbf{M}$ & $\operatorname{Tr}-1+$ & $1-3+$ & $\operatorname{Tr}-1+(3 / 5)$ & $3+$ & $1-3+$ \\
\hline & & & & $\begin{array}{ll}0 & (2 / 5)\end{array}$ & & \\
\hline$n=7$ & $\mathbf{F}$ & $\operatorname{Tr}-1+$ & $2-3+$ & $\operatorname{Tr}-1+(7 / 7)$ & $2-3+$ & $1-2+$ \\
\hline
\end{tabular}

* Intensity of basement membrane immunofluorescence was graded as: 0 , trace $(\mathrm{Tr}), 1+, 2+$, and $3+. \quad$ Serum from B-II-1, who developed antiGBM nephritis after renal transplantation from his HLA-identical sibling (B-II-2). § Results of staining urea-denatured skin are shown. EBM fluorescence was never seen with Goodpasture serum in the absence of urea denaturation. "Patients with gaps exhibited interrupted staining of EBM by FNS. II Indicates an affected family member.

of specimens that were not preincubated in urea. When GPS1 was incubated with urea-treated skin, binding of $\mathrm{IgG}$ to EBM was observed, in tissue from all members of the four kindreds studied except two (B-I-1 and C-II-2) and in tissue from 10 of 12 controls (Table I; Fig. 3 B). GPS2 stained EBM of three of seven controls, and nine of 11 kindred members (subjects B-I1 and C-I-2 showed no reactivity with GPS2). GPS3 reacted with five of six control specimens, and seven of 11 specimens from kindred members (B-I-1, B-II-4, C-I-1 and C-II-2 were negative). AGBM showed reactivity with EBM from six of six controls, and eight of 11 kindred members (B-I-1, B-II-4, and C-I-1 showed no reactivity). The EBM of all tissues studied reacted with anti-laminin and anti-type IV collagen, and this was not altered by urea preincubation (Fig. 3, $D$ and $E$ ).
Indirect immunofluorescence of the kidney was carried out in five (A-II-2, B-II-1, C-II-2, C-II-3, D-III-1) of the eight male patients with Alport-type familial nephritis (Fig. 5). FNS IgG did not bind to GBM, TBM, or Bowman's capsule of urea-treated or untreated tissues obtained from these patients, whereas brilliant staining of basement membranes was observed in normal tissue. Similar negative results were obtained when GPS1 was reacted with non-urea-treated kidney tissues of these five male patients. However, after urea preincubation, reactivity of GPS1 with TBM and Bowman's capsule was observed, while the GBM remained unreactive. When normal kidney was stained with GPS1, urea preincubation enhanced the intensity of GBM fluorescence, while TBM fluorescence became diffuse and Bowman's capsule also reacted positively. 

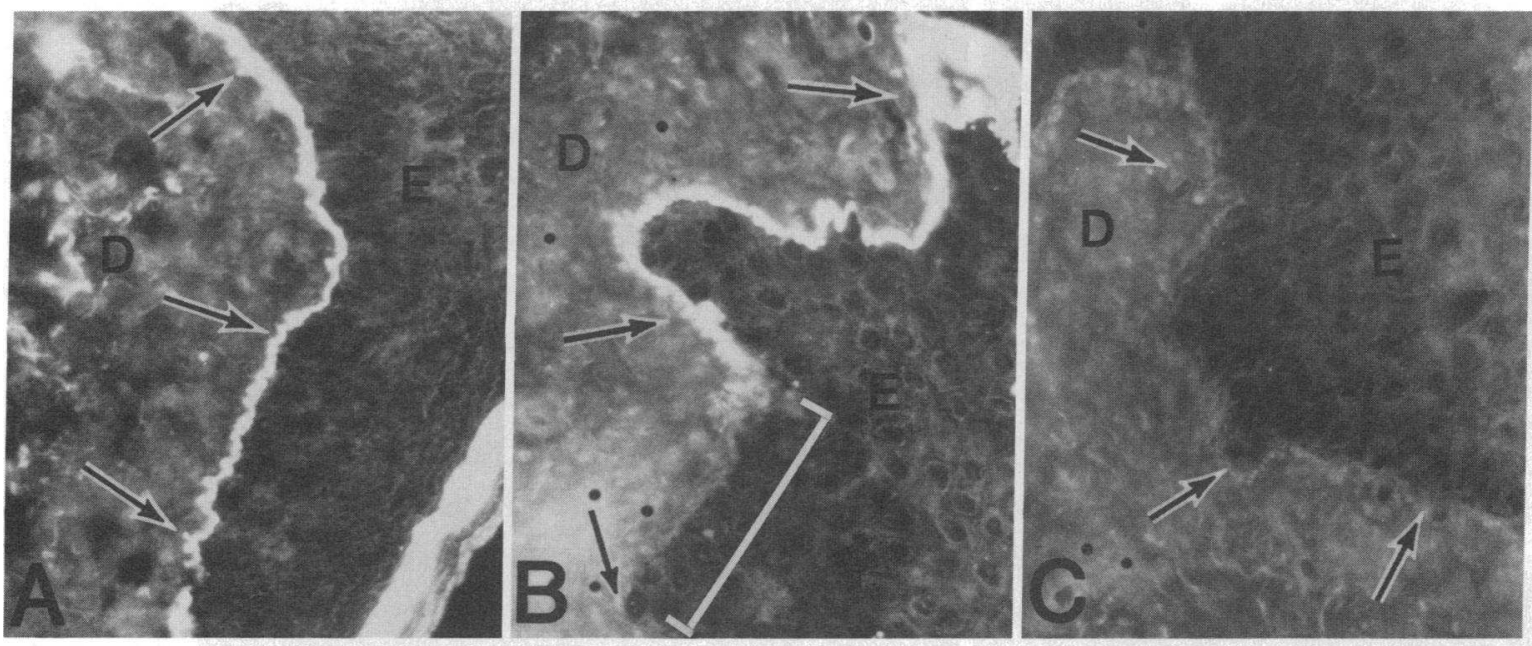

Figure 2. Reactivity of FNS with epidermal basement membranes of urea-denatured skin from three members of kindred D. (A) D-II-6: unaffected father of D-III-7. Note brilliant fluorescence of EBM (arrows) $(\times 475)$. (B) D-II-5: affected mother of D-III-7. Note discontinuity or gap in fluorescence of EBM. Nonreactive EBM is designated by bracket $(\times 390)$. (C) D-III-7: affected son. Note absence of EBM fluorescence (arrows) $(\times 370)$. Arrows, EBM; $E$, epidermis; D, dermis.

In three affected members of two kindreds ( $E$ and $F$ ) with BFH reactivity of FNS IgG and GPS1 IgG with EBM was similar to that found in normal individuals (Table I). Renal tissue from subject F-IV-1, a male with BFH, showed reactivity with GPS1 and FNS that was similar to that observed with normal renal tissue. In subject E-II-3, also a male, reactivity in GPS1 with GBM of non-urea-treated sections was diminished, but markedly enhanced by urea preincubation, and reactivity with FNS was similar to that observed in normal renal tissue.

Studies were also carried out in a family that was clinically similar to kindreds A-D but that is not included in the lettered pedigrees since electron microscopic studies of the kidney were not carried out. In contrast to the affected males in families A$D$, two affected males and one affected female in this family showed bright fluorescence of the EBM when FNS was reacted with urea-treated skin sections.

Characterization of anti-GBM sera. The results of SDSPAGE separation of collagenase-digested human GBM, and of immunoblotting of this material with the anti-GBM autoantibodies, are shown in Fig. 6. Collagenase-digests of salt and acid extracted normal human GBM contain, among other components, 43,000 to 54,000-mol-wt dimers and 24,000-mol-wt, 26,000-mol-wt, and 28,000-mol-wt monomers of type IV collagen NC1. By immunoblotting, GPS1, GPS2, GPS3 and AGBM identified $\mathrm{NC1}$ dimers as well as all three monomers; the most intense reactivity was observed with the 28,000 -mol-wt monomer. In contrast, FNS did not react with the 24,000-mol-wt and 28,000-mol-wt monomers, while strong reactivity was observed with the 26,000-mol-wt monomer and the dimers.

\section{Discussion}

Several groups of investigators have observed that in some patients with Alport-type familial nephritis (FN) the GBM does not bind human anti-GBM autoantibodies (10, 13-16). McCoy et al. (10) first described the development of anti-GBM antibodymediated glomerulonephritis (anti-GBM GN) in the renal al- lograft of a male with Alport-type FN. They demonstrated reactivity of the patient's anti-GBM serum with control kidneys, but not with his native kidneys or the kidneys of six other patients with Alport-type FN. It was suggested that the patient had developed antibodies to an antigen present in the donor kidney but absent in his own kidney and the kidneys of other patients with Alport-type FN. Subsequently, the development of antiGBM GN in the allografts of other Alport patients was reported $(11,12)$.

In the present study we have demonstrated that serum (designated FNS) obtained from a patient with Alport-type FN who developed post-transplant anti-GBM GN showed no reactivity with the patient's native kidney or with the kidneys of four other Alport males, although FNS contained antibody to normal GBM by indirect immunofluorescence and ELISA, and reacted with a 26,000-mol-wt monomer as well as dimers of the globular (NC1) domain of type IV collagen by immunoblots of one-dimensional gels. In addition, we have shown absence of binding of IgG antibody in FNS to epidermal basement membranes (EBM) of eight affected males in four kindreds with Alport-type FN, while in controls, FNS produced bright linear EBM fluorescence. Six of seven affected females in these four kindreds exhibited altered binding of FNS to EBM. One female with advanced renal insufficiency showed minimal reactivity of FNS with $\mathrm{EBM}$, while five others with asymptomatic microhematuria exhibited segmental loss of EBM reactivity. In nine unaffected male members of these kindreds, FNS stained EBM in a manner indistinguishable from that seen in normal individuals. These observations suggest that: (a) epidermal basement membranes, as well as renal basement membranes, display abnormal antigenic composition in individuals with Alport-type FN; and (b) members of Alport kindreds inherit this EBM abnormality in parallel with clinicopathologic evidence of renal and cochlear disease. Electron microscopic examination of skin specimens obtained from seven patients with Alport-type FN (four males, three females) has thus far failed to reveal definite ultrastructural abnormalities of EBM or dermal capillary basement membranes, 

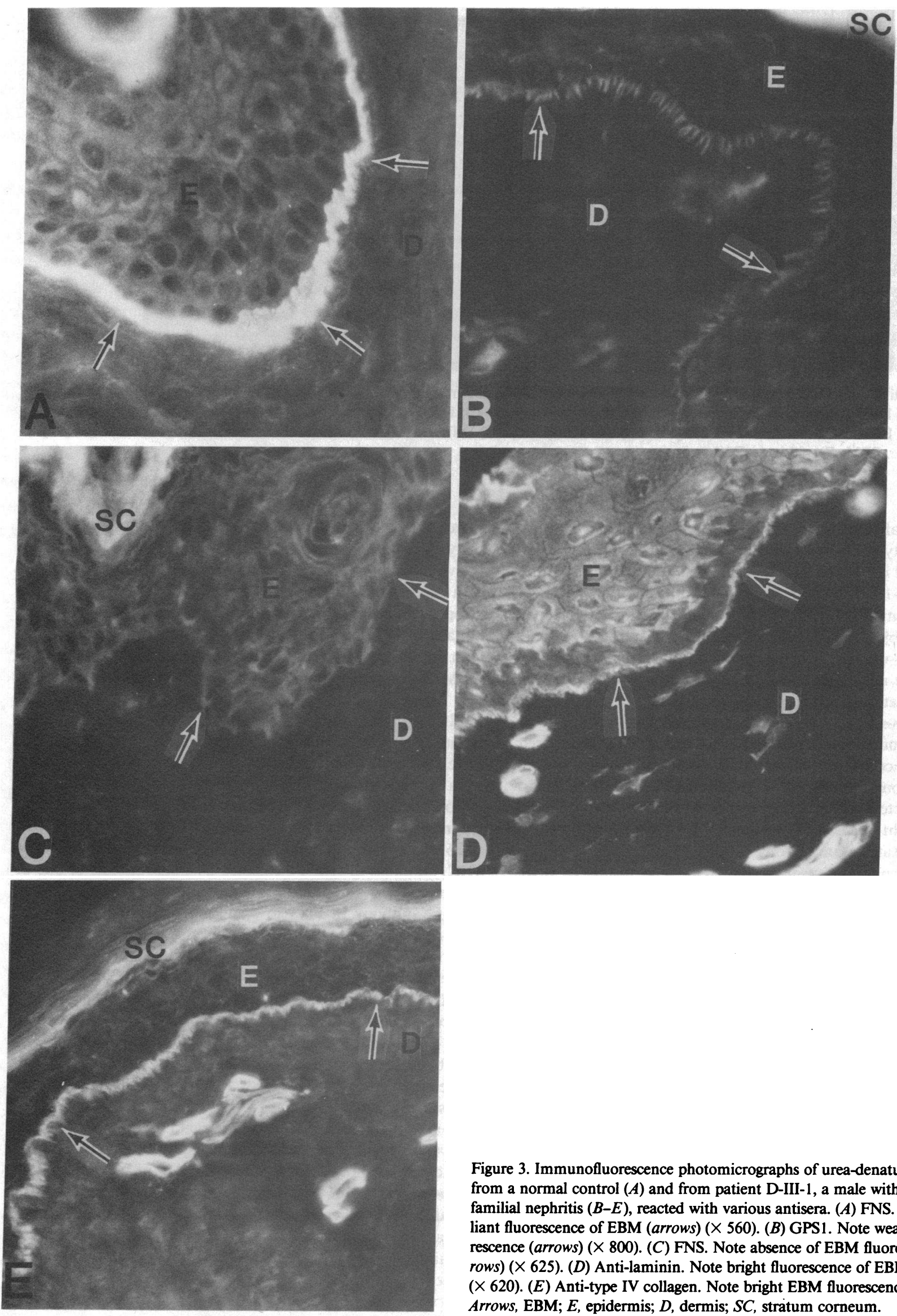

Figure 3. Immunofluorescence photomicrographs of urea-denatured skin from a normal control $(A)$ and from patient D-III-1, a male with Alport-type familial nephritis $(B-E)$, reacted with various antisera. $(A)$ FNS. Note brilliant fluorescence of EBM (arrows) $(\times 560)$. (B) GPS1. Note weak EBM fluorescence (arrows) $(\times 800)$. (C) FNS. Note absence of EBM fluorescence (arrows) $(\times 625)$. (D) Anti-laminin. Note bright fluorescence of EBM (arrows) $(\times 620)$. (E) Anti-type IV collagen. Note bright EBM fluorescence $(\times 540)$. Arrows, EBM; $E$, epidermis; $D$, dermis; $S C$, stratum corneum. 

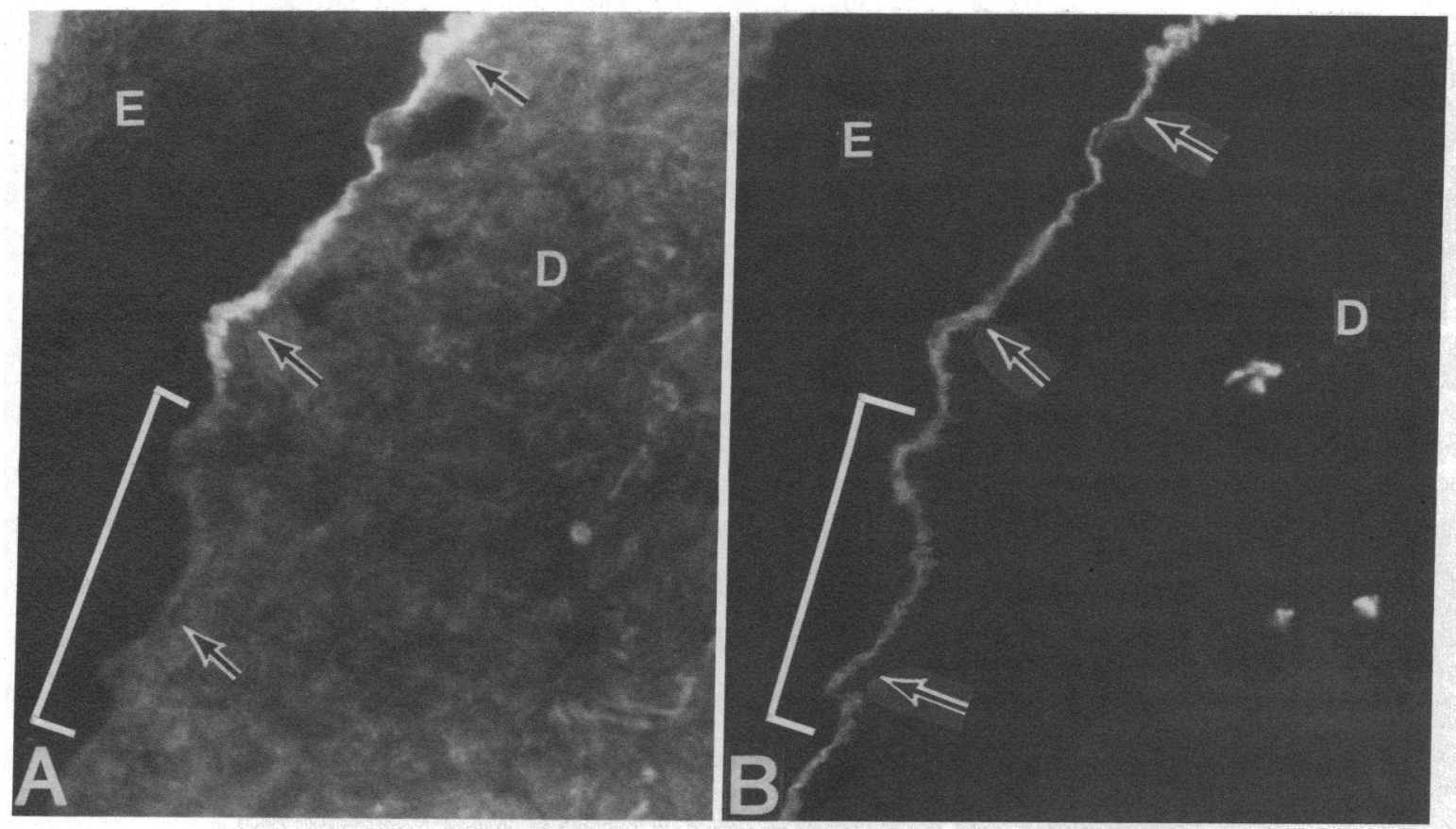

Figure 4. Immunofluorescence photomicrographs of urea-denatured skin from patient D-II-5, a female patient with Alport-type familial nephritis. The same section was stained sequentially with FNS and FITC-labeled rabbit anti-human $\operatorname{IgG}(A)$ followed by mouse monoclonal anti-human type IV collagen and rhodamine-labeled goat anti-mouse IgG $(B)$. Note the absence of fluorescence with FNS in a segment of the EBM in $A$ (bracket). This same region, however, reacts with anti-type IV collagen as shown in $B$ (bracket). Arrows, EBM; $E$, epidermis; $D$, dermis; $S C$, stratum corneum.

although these patients exhibited altered reactivity with FNS as described herein (H. Tochimaru, C. Kashtan, and R. L. Vernier, unpublished observations).

Sera from three patients with Goodpasture's syndrome (GPS), i.e., anti-GBM GN associated with pulmonary hemorrhage, and serum from a patient with isolated anti-GBM GN, gave results different from those seen with FNS. In skin, reactivity of these sera with EBM was seen only in urea-treated sections; controls as well as affected and unaffected members of Alport kindreds demonstrated similar GPS binding to EBM. While GPS1 did not stain renal basement membranes of routinely processed sections of kidney from five males with Alport-type FN, it reacted with the TBM and Bowman's capsule after denaturation of tissue sections in acid-urea. In contrast, FNS lacked reactivity with all renal basement membranes in these patients despite urea-preincubation.

The differences observed among the Goodpasture sera in their reactivity with EBM may have resulted from several variables: variation in the quantities of anti-GBM antibodies; the presence of antibodies with differing specificities to epitopes which vary in their expression in EBM; and heterogeneity in the antigenic composition of EBM in normal individuals related to genetic influences. In any case, among the anti-GBM antisera studied, only FNS distinguished between EBM of individuals with Alport-type FN and EBM of normal subjects.

The differences in reactivity of the GPS and FNS with ureatreated tissues of patients with Alport-type FN suggest that the antisera are directed against different epitopes. The lack of GPS1 binding to GBM of urea-treated kidney tissues of male patients with Alport-type FN may reflect absence of the relevant epitope from Alport GBM, or alteration in the structure of Alport GBM such that the relevant epitope remains hidden despite urea denaturation. The presence in GPS1 of a distinct antibody directed against an epitope located in TBM and BC but not GBM could account for the failure of urea denaturation to expose an antigen in Alport GBM that appears to be present in the TBM and BC.

Isolated basement membranes can be partially solubilized by urea, via disruption of hydrophobic and hydrogen bonds (30). Prior immunohistochemical studies from this laboratory have shown that preincubation of tissue sections in acid-urea permits binding of anti-GBM antibodies to antigenic determinants that are concealed under native conditions (17). In an analysis of the binding characteristics of 10 anti-GBM sera, it was found that only four reacted with the GBM of fetal/newborn kidneys, whereas binding of the remaining six could be demonstrated only after acid-urea denaturation (12). Similarly, urea denaturation exposed antigenic sites for reactivity with anti-GBM antibodies in basement membranes of lung, skin, and placenta (17). In addition, Odermatt and colleagues (31) have shown that urea denaturation uncovers renal basement membrane epitopes reactive with monoclonal antibodies to type IV collagen. These findings further support the notion that antigenic structures normally hidden within basement membranes can be "unmasked" by exposure of tissues to acid solutions of urea.

Recent studies suggest a mechanism by which epitopes reactive with Goodpasture sera might be unmasked in basement membranes. Weislander and colleagues $(18,32)$ demonstrated that the Goodpasture epitope is located in the noncollagenous (NC1) globular domain at the carboxy-terminal end of type IV collagen. This globular region exists as a hexamer that is re- 

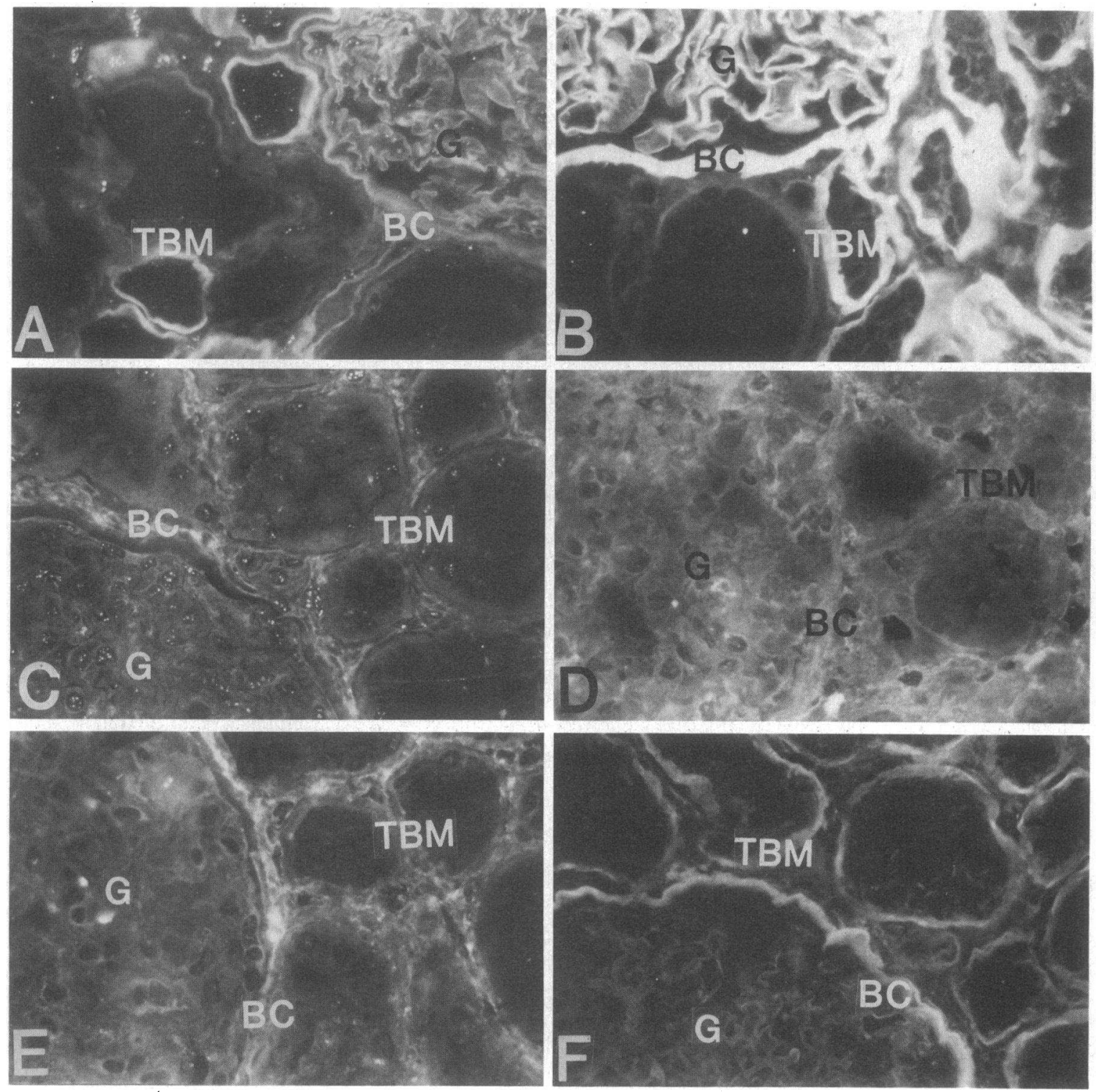

Figure 5. Immunofluorescence photomicrographs of normal kidney $(A, B)$ and renal tissue from patient C-II-2, a male with Alport-type familial nephritis $(C-F)$. Sections were stained with FNS or GPSI and FITC-labeled rabbit anti-human IgG. $(A)$ Normal tissue (nondenatured) stained with FNS. Note linear fluorescence of GBM, BC, and occasional TBM $(\times 370)$. $(B)$ Normal tissue (urea-denatured) stained with FNS. Note enhanced fluorescence of GBM, BC, and TBM $(\times 375)$. (C) Alport-type FN (nondenatured) stained with FNS. Note absence of GBM, TBM, or BC reactivity $(\times 380)$. (D) Alport-type FN (urea-denatured) stained with FNS. Note absence of GBM, TBM, or BC reactivity $(\times 380)$. $(E)$ Alport-type FN (nondenatured) stained with GPS1. Note absence of GBM, TBM, or BC reactivity $(\times 390)$. $(F)$ Alport-type FN (urea-denatured) stained with GPS1. Note fluorescence of TBM and BC but not GBM $(\times 380)$. $G$, glomerulus; $B C$, Bowman's capsule.

versibly dissociated by denaturation in $6 \mathrm{M}$ guanidine or in acidic solutions, and under these conditions there is enhanced reactivity with Goodpasture antibody (33). These findings suggest that the Goodpasture epitope is for the most part sequestered within the hexameric globular domain of type IV collagen. The variable reactivity of basement membranes of different tissues with human anti-GBM sera observed by immunohistochemical techniques (17) may be a consequence of variation in the tertiary structure of the $\mathrm{NCl}$ hexamer in situ, with some tissues containing more exposed Goodpasture epitopes than others. Treatment of tissues with acid-urea likely causes dissociation within the hexameric globular domain with further exposure of the reactive epitope(s) by disruption of hydrophobic interactions as well as ionic bonds.

We have demonstrated that, like GPS, FNS reacts with monomeric and dimeric components of $\mathrm{NCl}$. However, reactivity of FNS appears to be restricted to the 26,000-mol-wt monomer, while GPS reacts most strongly with the 28,000 -mol-wt monomer in addition to the 24,000- and 26,000-mol-wt components. This finding concurs with our previous report (20) that FNS failed to react with very cationic components of $\sim 27,000 \mathrm{~mol}$ wt (isolated by two-dimensional gel electrophoresis) that were identified by nine other human anti-GBM sera. These observations constitute additional evidence that FNS and GPS are 


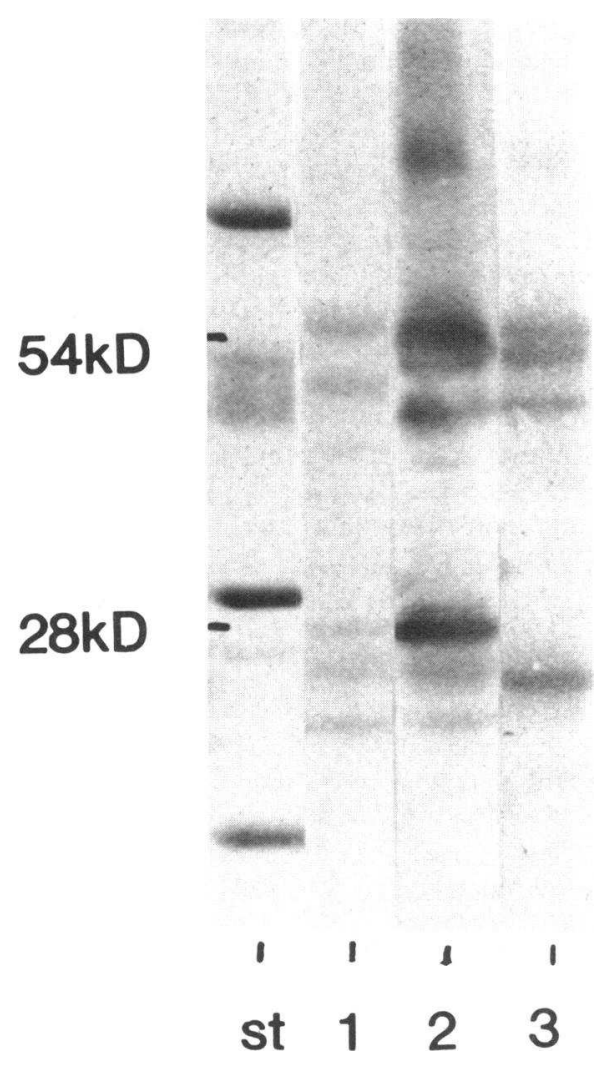

Figure 6. Coomassie stain and immunoblots of collagenase digests of salt and acid extracted normal human glomerular basement membranes, separated on 8-18\% SDS-PAGE gels. Lane st, Coomassie stain of low molecular weight standards (BSA, $68,000 \mathrm{~mol} \mathrm{wt}$; ovalbumin, 43,000 mol wt; carbonic anhydrase, 29,000 mol wt; and lactalbumin, $14,000 \mathrm{~mol} \mathrm{wt}$ ). Lane 1, Coomassie stain of normal human GBM. Note proteins of $\sim 43,000$ to $54,000 \mathrm{~mol}$ wt (type IV collagen $\mathrm{NCl}$ dimers) and proteins of $24,000,26,000$, and $28,000 \mathrm{~mol}$ wt (type IV collagen NC1 monomers). Lane 2, Immunoblot of normal human GBM, using Goodpasture serum (GPS1). Note reactivity with $\mathrm{NCl}$ dimers $(43,000$ to $54,000 \mathrm{~mol} \mathrm{wt})$, and monomers $(24,000,26,000$, and $28,000 \mathrm{~mol} w \mathrm{wt}$ ). The 28,000-mol-wt monomer exhibits the most intense reactivity with GPS1. The same pattern was seen with GPS2, GPS3, and AGBM. Reactivity with higher molecular weight components $(\sim 75,000 \mathrm{~mol} w \mathrm{wt})$ is noted. These may represent multimeric components. Lane 3, Immunoblot of normal human GBM, using anti-GBM serum (FNS) from an Alport patient (patient B-II-1 in Fig. 1). While reactivity with the 43,000 - to 54,000 -mol-wt $\mathrm{NCl}$ dimers is observed, FNS identifies only the 26,000-mol-wt monomer, and fails to react with the 24,000 - and 28,000 -mol wt monomers.

directed against different epitopes on the $\mathrm{NC1}$ domain of type IV collagen.

The genetics of Alport-type FN has been the subject of extensive debate; no single mechanism appears to explain inheritance patterns in all families. There is increasing acceptance of the hypothesis that Alport-type FN is a genetically heterogeneous disorder, with autosomal dominant, X-linked dominant, and autosomal recessive forms (34). The kindreds presented in this paper exhibit inheritance patterns consistent with an X-linked dominant mechanism, although other modes of inheritance have not been excluded. The pattern of FNS reactivity with EBM found in kindreds $A-D$ is also consistent with $\mathrm{X}$-linked dominant inheritance. Males in these kindreds, if affected, displayed ap- parent absence of the antigen reactive with FNS, while unaffected male relatives were indistinguishable from controls. Affected females had diminished binding of FNS by EBM, and in five of the females a remarkably segmental EBM staining pattern was observed. In these female patients, we postulate that FNS-reactive segments of EBM are synthesized by clonally-derived basal keratinocytes in which the defective $\mathrm{X}$ chromosome has been randomly inactivated. Conversely, where the EBM fails to bind FNS, the normal X chromosome has been inactivated. A similar $\mathrm{X}$-linked mosaicism in epidermal structures has been described classically in the calico cat and in humans with anhydrotic ectodermal dysplasia (35). The extent of EBM nonreactivity (and by extension, the severity of the disease in the affected female) would then depend on the proportion of basement-membrane producing cells in which the abnormal $\mathrm{X}$ chromosome was active. O'Neill and colleagues (36) previously proposed this mechanism to explain the genetics of Alport-type FN. We have previously presented immunofluorescence data on renal tissue that was consistent with this hypothesis (15).

Despite having a disease indistinguishable by clinical criteria from that manifested by affected males in families A-D, the two affected males in an additional family exhibited normal staining of EBM by FNS. Habib et al. (16), have reported Alport patients, including males, in whom GBM reacted normally with antiGBM sera. These findings suggest that Alport-type FN may consist of subtypes distinguishable by their antigenic phenotypes.

Our finding of an antigenic abnormality of EBM in some Alport kindreds is consistent with the hypothesis, put forth by Spear (37) in 1973, that a generalized abnormality of a common basement membrane constituent accounts for the clinical and pathological characteristics of Alport-type FN. Our observations suggest that the abnormality lies in type IV collagen.

\section{Acknowledgments}

The authors wish to thank the members of families A-F for participating in this study. Dr. Michelle Lopez de Diaz and Mrs. Vivian Diaz kindly obtained some of the tissues we studied. Ms. Crystal Blocher, Ms. Kathy Divine, Ms. Kim Pinkham and Ms. LuAnn Anderson performed the immunohistological studies, and Ms. Lore Lang prepared the tissues for staining. Ms. Tami Petersen and Ms. Cindy Dawis typed the manuscript. Mr. Marshall Hoff and Ms. Kathy DeWell prepared the illustrations.

This work was supported by National Institutes of Health grants AI-10704, AM-25518, and AM-26149, and grants from the Viking Children's Fund, National Diabetes Research Interchange, University of Minnesota Graduate School, and the Minnesota Medical Foundătion.

\section{References}

1. Spear, G. S., and R. J. Slusser. 1972. Alport's syndrome: emphasizing electron microscopic studies of the glomerulus. Am. J. Pathol. 69: 213-224.

2. Hinglais, N., J.-P. Grunfeld, and E. Bois. 1972. Characteristic ultrastructural lesion of the glomerular basement membrane in progressive hereditary nephritis (Alport's syndrome). Lab. Invest. 27:473487.

3. Rumpelt, H. J., K. H. Langer, K. Scharer, E. Straub, and W. Theones. 1974. Split and extremely thin glomerular basement membranes in hereditary nephropathy (Alport's syndrome). Virchows Arch. A Pathol. Anat. Histol. 364:225-233.

4. Rumpelt, H. J. 1980. Hereditary nephropathy (Alport syndrome): correlation of clinical data with glomerular basement membrane alterations. Clin. Nephrol. 13:203-207.

5. Yoshikawa, N., A. H. Cameron, and R. H. R. White. 1981. The glomerular basal lamina in hereditary nephritis. J. Pathol. 135:199-209. 
6. Kohaut, E. C., D. B. Singer, B. K. Nevels, and L. L. Hill. 1976. The specificity of split renal membranes in hereditary nephritis. Arch. Pathol. Lab. Med. 100:475-479.

7. Gaboardi, F., A. Edefonti, E. Imbasciati, A. Tarantino, M. J. Mihatsch, and H. V. Zollinger. 1974. Alport's syndrome (progressive hereditary nephritis). Clin. Nephrol. 2:143-156.

8. Yoshikowa, N., R. H. R. White, and A. H. Cameron. 1982. Familial hematuria: clinicopathological correlations. Clin. Nephrol. 17:172-182.

9. Gubler, M. C., G. Gonzales-Burchard, C. Monnier, and R. Habib. 1976. Alport's syndrome: natural history and ultrastructural lesions of glomerular and tubular basement membranes. Contrib. Nephrol. 2:163169.

10. McCoy, R. C., H. K. Johnson, W. J. Stone, and C. B. Wilson. 1982. Absence of nephritogenic GBM antigen(s) in some patients with hereditary nephritis. Kidney Int. 21:642-652.

11. Milliner, D. S., A. M. Pierides, and K. E. Holley. 1982. Renal transplantation in Alport's syndrome: anti-glomerular basement membrane glomerulonephritis in the allograft. Mayo Clin. Proc. 57:35-43.

12. Jeraj, K., A. J. Fish, K. Yoshioka, and A. F. Michael. 1984. Development and heterogeneity of antigens in the immature nephron. Reactivity with human antiglomerular basement membrane autoantibodies. Am. J. Pathol. 117:180-183.

13. Olson, D. L., S. K. Anand, B. H. Landing, E. Heuser, C. M. Grushkin, and E. Lieberman. 1980. Diagnosis of hereditary nephritis by failure of glomeruli to bind anti-glomerular basement membrane antibodies. J. Pediatr. 96:697-699.

14. Jenis, E. H., J. E. Valeski, and P. L. Calcagno. 1981. Variability of anti-GBM binding in hereditary nephritis. Clin. Nephrol. 15:111-114.

15. Jeraj, K., Y. Kim, R. L. Vernier, A. J. Fish, and A. F. Michael. 1983. Absence of Goodpasture's antigen in male patients with familial nephritis. Am. J. Kidney Dis. 11:626-629.

16. Habib, R., M. C. Gubler, N. Hinglais, L. H. Noel, D. Droz, M. Levy, P. Mahieu, J. M. Foidart, O. Perrin, E. Bois, and J.-P. Grunfeld. 1982. Alport's syndrome: experience at Hopital Necker. Kidney Int. 21: S20-S28.

17. Yoshioka, K., A. F. Michael, J. Velosa, and A. J. Fish. 1985. Detection of hidden nephritogenic antigen determinants in human renal and non-renal basement membranes. Am. J. Pathol. 121:156-165.

18. Wieslander, J., J. F. Barr, R. J. Butkowski, S. J. Edwards, P. Bygren, D. Heinegard, and B. G. Hudson. 1984. Goodpasture's antigen of the glomerular basement membrane: localization to noncollagenous regions of type IV collagen. Proc. Natl. Acad. Sci. USA. 81:3838-3842.

19. Scheinman, J. I., and C. Tsai. 1984. Monoclonal antibody to type IV collagen with selective basement membrane localization. Lab. Invest. 50:101-112.

20. Yoshioka, K., M. Kleppel, and A. J. Fish. 1985. Analysis of nephritogenic antigens in human glomerular basement membrane by two-dimensional gel electrophoresis. J. Immunol. 134:3831-3837.

21. Fish, A. J., M. Kleppel, K. Jeraj, and A. F. Michael. 1985. Enzyme immunoassay of anti-glomerular basement membrane antibodies. J. Lab. Clin. Med. 105:700-705.
22. Westberg, N. G., and A. F. Michael. 1970. Human glomerular basement membrane. Separation and composition. Biochemistry. 9: 3837-3846.

23. Timpl, R., H. Wiedemann, V. Van Delden, H. Furthmayr, and K. Kuhn. 1981. A network model for the organization of type IV collagen molecules in basement membranes. Eur. J. Biochem. 120:203-211.

24. Laemmli, U. K., 1970. Cleavage of structural proteins during assembly of the head of bacteriophage T4. Nature (Lond.). 227:680-685.

25. Burnette, W. N. 1981. "Western blotting": Electrophoretic transfer of proteins from sodium dodecyl sulfate-polyacrylamide gels to unmodified nitrocellulose and radiographic detection with antibody and radioiodinated protein A. Anal. Biochem. 112:195-203.

26. Bittner, M., P. Kupferer, and C. F. Morris. 1980. Electrophoretic transfer of proteins and nucleic acids from slab gels to diazobenzyloxymethyl cellulose or nitrocellulose sheets. Anal. Biochem. 102:459-471.

27. Brandtzaeg, P. 1976. Studies on J chain and binding site for secretory component in circulating B cells. II. The cytoplasm. Clin. Exp. Immunol. 25:50-58.

28. Bene, M. C., B. Faure, B. Hurault de Ligny, M. Kessler, and J. Duheille. 1983. Immunoglobulin A nephropathy. Quantitative immunohistomorphometry of the tonsillar plasma cells. Evidences and inversion of the immunoglobulin $\mathrm{G}$ secreting cell balance. J. Clin. Invest. 71: 1342-1347.

29. Platt, J. L., and A. F. Michael. 1983. Retardation of fading and enhancement of intensity of fluorescence by p-phenylenediamine. $J$. Histochem. Cytochem. 31:840-842.

30. Kefalides, N. A. 1972. Biochemical studies of the glomerular basement membrane in the normal kidney. Adv. Nephrol. 2:3-24.

31. Odermatt, B. F., A. B. Lang, J. R. Ruttner, K. H. Winterhalter, and B. Trueb. 1984. Monoclonal antibodies to human type IV collagen: useful reagents to demonstrate the heterotrimeric nature of the molecule. Proc. Natl. Acad. Sci. USA. 81:7343-7347.

32. Butkowski, R. J., J. Wieslander, B. J. Wisdom, J. F. Barr, M. E. Noelken, and B. G. Hudson. 1985. Properties of the globular domain of type IV collagen and its relationship to Goodpasture antigen. J. Biol. Chem. 260:3739-3747.

33. Weislander, J., J. Langveld, R. Butkowski, M. Jodlowski, M. Noelken, and B. G. Hudson. 1985. Physical and immunochemical studies of the globular domain of type IV collagen: cryptic properties of the Goodpasture antigen. J. Biol. Chem. 260:8564-8570.

34. Feingold, J., E. Bois, A. Chompret, M. Broyer, M. E. Gubler, and J.-P. Grunfeld. 1985. Genetic heterogeneity of Alport syndrome. Kidney Int. 27:672-677.

35. Mange, A. P., and E. J. Mange. 1980. Genetics: Human Aspects. Saunders College, Philadelphia. 147-153.

36. O'Neill, W. M., C. L. Atkin, and H. A. Bloomer. 1978. Hereditary nephritis: a reexamination of its clinical and genetic features. Ann. Intern. Med. 88:176-182.

37. Spear, G. S. 1973. Alport's syndrome: consideration of pathogenesis. Clin. Nephrol. 1:336-337. 